\title{
Anxiety and depression in an older research population and their impact on clinical outcomes in a randomised controlled trial
}

\section{S J Allsup, M A Gosney}

See end of article for authors' affiliations

Correspondence to: Dr S Allsup, Department of Geriatric Medicine, University of Liverpool, University Clinical Department, Duncan Building, Daulby Street, Liverpool L69 3GA, UK flutrial@liv.ac.uk

Submitted 30 April 2002 Accepted 22 July 2002

\begin{abstract}
Introduction: Anxiety and depression are common in older people living in the community. The aim of the study was to investigate their impact on clinical outcomes during a randomised controlled trial investigating the cost benefits of influenza vaccination in fit and healthy, independent living 65-74 year olds.

Subjects and methods: A total of 729 people were recruited. Participants completed the hospital anxiety and depression scale (HADS) and EuroQol EQ-5D quality of life questionnaire immediately before receiving vaccination and every two months for the next six months after this. Side effects three days after vaccination and Barthel score at baseline were also recorded.

Results: At baseline the prevalence of "definite" anxiety in this sample (HADS score $\geqslant 11$ ) was $4 \%$ and $1.2 \%$ of individuals had definite depression (HADS score $\geqslant 11$ ). Individuals with anxiety or depression (HADS score $\geqslant 8$ ) were more likely to complain of systemic side effects after vaccination and have a lower Barthel index score $(p<0.001)$. Quality of life as measured by the EQ-5D visual analogue scale was reduced $(p<0.001)$ at all time periods in those individuals with both anxiety and depression (HADS score $\geqslant 8$ on both scales).

Conclusion: Although the prevalence of anxiety and depression in this sample was low, people with anxiety or depression were more likely to suffer from perceived side effects after influenza vaccine and have a lower Barthel and EQ-5D visual analogue score. In future studies the effect of anxiety and depression on older participants should be remembered and care taken to ensure that they do not affect results more than the intervention under study.
\end{abstract}

A nxiety and depression are common in the community. A recent systematic review of 34 community based studies found an average prevalence for depressive syndromes of $13.5 \%$ in adults aged 55 years and over, although the reported prevalence rate was found to vary enormously between $0.4 \%$ and $35 \% .{ }^{1}$ The prevalence of anxiety disorders in older people living in the community has been estimated to be $15 \% .^{2}$ Anxiety and depression have been shown to have a negative effect on quality of life. ${ }^{34}$ The results and conclusions of a clinical trial conducted in the community may therefore be affected if people consenting for the study have anxiety or depressive disorders-for example, by influencing the drop out rate or affecting the side effect profile of a new treatment. As part of a study to determine the cost benefits of influenza vaccination in fit and healthy people aged 65-74 years of age, we monitored anxiety and depressive symptoms for six months after vaccination using the hospital anxiety and depression scale (HADS). We also examined the effect that anxiety or depression had on systemic side effects after vaccination, quality of life as measured by the EuroQol EQ-5D, functional ability as measured by the Barthel index, and return of postal questionnaires.

\section{SUBJECTS AND METHODS}

Altogether 729 healthy individuals (388 males/341 females; median age 68.9 years) not previously requiring influenza vaccination as defined by the 1998 United Kingdom Chief Medical Officers' guidelines ${ }^{5}$ were recruited to join the study starting October 1999. Individuals were block randomised in a ratio 3:1 to receive either influenza vaccine or placebo. Immediately before receiving vaccination (which was given by researchers based at the patient's local general practice) and for every two months for the next six months (that is December, February, April) as a postal questionnaire, all individuals were asked to complete the HADS and EuroQol EQ-5D questionnaire. The HADS consists of 14 items on two subscales (seven anxiety and seven depression) and is self administered. Ratings are made on four point scales representing the degree of distress during the previous week. The two scales are then scored separately. A score of 7 or less indicates non-cases, 8-10 doubtful cases, and $11+$ definite cases. In the original paper, the authors argued that "should the researcher require inclusion of all possible cases, that is, a low proportion of false negatives", then a score of 8 and over should be used, and "where the research requires the inclusion of only those patients who have a high probability of suffering from the mood disorder, that is a low proportion of false positives", then a score of 11 and over should be used. ${ }^{6}$ Both subscales have been shown in different studies to have good reliability and validity when used as a psychological screening tool in a hospital type setting and are sensitive to changes in patients' emotional state in longitudinal assessments. ${ }^{7}$ It does, however, appear to be less effective as a research tool in detecting the presence of a major depressive disorder. ${ }^{8}$

The EuroQol EQ-5D questionnaire was developed as a practical way of measuring the health of a population and enables a self description of current health related quality of life to be easily recorded. Health states are measured in the form of a self reported description using a five dimensional classification and converted to a weighted score. Alternatively a

Abbreviations: HADS, hospital anxiety and depression scale; EuroQol $E Q-5 D$, quality of life questionnaire; $M$, month 
Table 1 Number (\%) of individuals in different threshold categories for anxiety and depression at baseline

\begin{tabular}{lllll}
\hline & \multicolumn{2}{l}{ Depression subscale } & \\
\cline { 2 - 5 } Anxiety subscale & Non-case $(0-7)$ & $\begin{array}{l}\text { Doubtful case } \\
(8-10)\end{array}$ & $\begin{array}{l}\text { Definite case } \\
(\geqslant 11)\end{array}$ & Total \\
\hline Non-case $(0-7)$ & $605(87.1)$ & $10(1.4)$ & $3(0.4)$ & $618(88.9)$ \\
Doubtful case (8-10) & $40(5.8)$ & $9(1.3)$ & 0 & $49(7.1)$ \\
Definite case ( $\geqslant 11)$ & $17(2.4)$ & $6(0.9)$ & $5(0.7)$ & $28(4.0)$ \\
Total & $662(95.3)$ & $25(3.6)$ & $8(1.2)$ & $695(100)$ \\
\hline
\end{tabular}

visual analogue "thermometer" scale can be used, where 100 represents the best imaginable health state and 0 the worst.

At the time of vaccination, participants were also given a closed questionnaire asking specifically about certain side effects (sore arm, cough, fever, aching limbs, malaise, rash, headache, sore throat, runny nose) with instructions to complete and return it in a pre-paid envelope to the researchers after three days. A Barthel functional health assessment index was also self completed immediately before vaccination.

Full approval for the study was obtained from the local research and ethics committee and informed consent was obtained from all individuals before entry into the study.

\section{Statistical analysis}

Differences between proportions were analysed using the $\chi^{2}$ test (correction factor applied). Differences between means were analysed using Mann-Whitney U test. Change in anxiety and depression over time was analysed using a repeated measure analysis of variance (general linear model). All data were held in Microsoft Access 97 and analysed using SPSS v 10 .

\section{RESULTS \\ Prevalence of anxiety and depression in the study population}

A total of 695 (95\%) individuals completed all 14 questions of the HADS, immediately before receiving influenza vaccination (table 1). The remaining 34 individuals failed to answer one or more questions on either scale and were thus not included in table 1 .

The number of individuals in different threshold ranges for both the anxiety and depression scale is shown in table 1 . Altogether 605 individuals (87.1\%) had no significant anxiety and depression. Using a threshold of 8 or above, 90 (12.9\%) individuals would be classified as suffering from either anxiety or depression (the sum of all "definite" and "doubtful" cases). Similarly, using a threshold of $11+, 31$ $(4.5 \%)$ individuals would be classified as suffering from either anxiety or depression.

At baseline, $28(4 \%)$ individuals had definite anxiety (11 males/17 females) and eight (1.2\%) had definite depression (four males/four females). Of the 28 individuals with definite anxiety, six (21.4\%) remained definitely anxious at each two month monitoring period. Of the eight individuals with definite depression, only one person remained definitely depressed for the whole six month monitoring period.

Figures 1 and 2 illustrate the mean variation in anxiety and depression scoring over the six month monitoring period. Five hundred and fifty two individuals returned and fully completed the anxiety subscale of the HADS questionnaire for every two month period. The mean anxiety score for each period was: month $(M) \quad 0=3.52, M 2=3.67$, M4=3.64, M6=3.51. There were no significant differences between these means $(\mathrm{p}=0.265)$.

Five hundred and twenty eight individuals returned, fully completed, the depression subscale of the HADS questionnaire for every two month period. The mean depression score

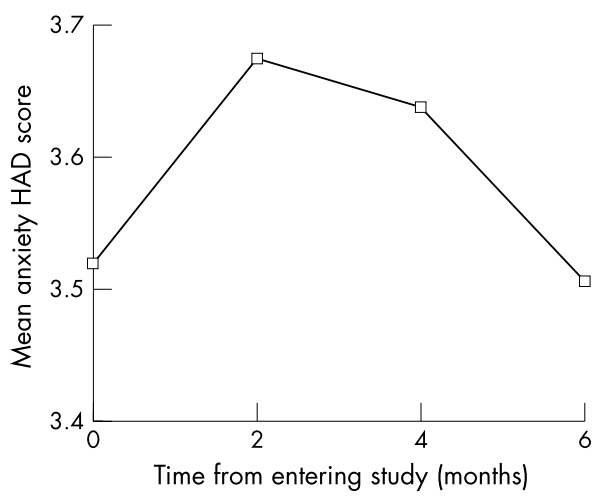

Figure 1 Mean anxiety over six months.

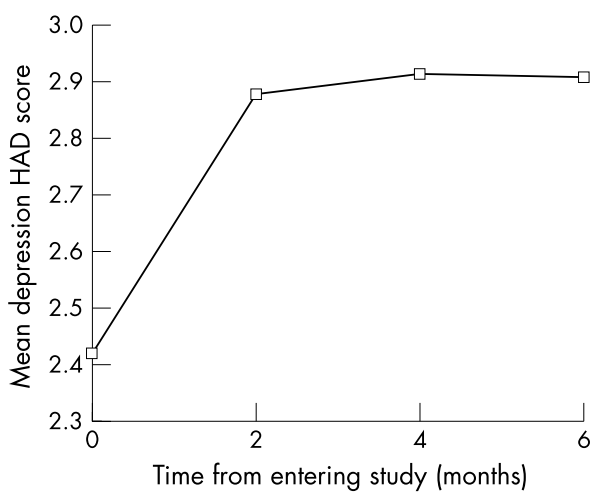

Figure 2 Mean depression over six months.

for each period was: $M 0=2.42, \quad M 2=2.88, \quad M 4=2.92$, $\mathrm{M} 6=2.91$. There was a significant increase $(\mathrm{p}<0.001)$ in mean score from baseline (2.42) to two months (2.88), but no significant change in mean score was seen after two months.

Barthel scores at baseline and anxiety and depression score

Altogether 722 Barthel indexes were fully completed at baseline. The mean (SD) Barthel score for individuals scoring between 0 and 7 on the anxiety subscale was 99 (4.1) compared with a mean score of 94.4 (9.5) for individuals scoring 8 or more $(\mathrm{p}<0.001)$.

The mean (SD) Barthel score for individuals scoring between 0 and 7 on the depression subscale was 98.8 (4.5) compared with a mean score of 92.9 (11.0) for individuals scoring 8 or more $(\mathrm{p}<0.001)$.

\section{Quality of life}

Table 2 illustrates the association between mean quality of life score using the EQ-5D visual analogue "thermometer" scale and scoring on both anxiety and depression scales when using 8 as the threshold value at each two month monitoring period. 
Table 2 Mean EQ-5D self rated health state using the visual analogue (VA) "thermometer" scale recorded at two monthly intervals

\begin{tabular}{llll}
\hline $\begin{array}{l}\text { Time period from } \\
\text { study injection } \\
\text { (months) }\end{array}$ & \multicolumn{2}{l}{ Mean (SD) EQ-5D VA score* } & \\
\cline { 2 - 3 } & $0-7$ & $8-21$ & p Value \\
\hline 0 & $84.5(14.4)$ & $63.1(17.4)$ & $<0.001 \dagger$ \\
2 & $83.2(12.7)$ & $58.9(18.4)$ & $<0.001 \dagger$ \\
4 & $83.7(11.9)$ & $57.6(19.9)$ & $<0.001 \dagger$ \\
6 & $84.0(12.7)$ & $53.3(19.9)$ & $<0.001 \dagger$ \\
\hline
\end{tabular}

*Individuals scoring 0-7 or 8-21 on both anxiety and depression subscales.

†Mann-Whitney U test

Table 3 Anxiety and systemic side effects three days after study injection

Side effects
Yes No Total

Anxiety score $\geqslant 8$

Yes

No

Total 47 209 32 414 79 256

$446 \quad 702$

Pearson's $\chi^{2}$ correction factor applied $=19.267 ; \mathrm{p}<0.001$

Table 4 Depression and systemic side effects three days after study injection

\begin{tabular}{llll}
\hline & \multicolumn{2}{l}{ Side effects } & \\
\cline { 2 - 3 } & Yes & No & Total \\
\hline Depression score $\geqslant 8$ & & & \\
Yes & 24 & 9 & 33 \\
No & 236 & 438 & 674 \\
Total & 260 & 447 & 707 \\
\hline
\end{tabular}

Pearson's $\chi^{2}$ correction factor applied $=17.656 ; p<0.001$.

There was a significant difference in mean EQ-5D scores $(\mathrm{p}<0.001)$ at every two month period between individuals scoring 8 or more on both anxiety and depression subscales and those scoring 7 or less.

\section{Side effects and anxiety and depression score.}

Tables 3 and 4 illustrate the number of individuals with anxiety or depression (HADS score $\geqslant 8$ ) that complained of one or more systemic side effects after influenza vaccine. Seven hundred and two people completed all seven questions on the anxiety subscale and have been included in table 3. Seven hundred and seven people completed all seven questions on the depression subscale and have been included in table 4 .

Fifty nine per cent of people with anxiety (HADS score $\geqslant 8$ ) complained of one or more systemic side effects compared with $34 \%$ of people with no anxiety (HADS score $\leqslant 7$ ); $\mathrm{p}<0.001$. Seventy three per cent of people with depression (HADS score $\geqslant 8$ ) complained of one or more systemic side effects compared with $35 \%$ of people with no depression (HADS score $\leqslant 7$ ); $\mathrm{p}<0.001$.

\section{Non-return of questionnaires and anxiety and depression score}

There was no association between baseline anxiety or depression score and non-return of questionnaires at any time period.

\section{DISCUSSION}

The prevalence of both anxiety and depression was low in this sample of individuals. Comparability with other studies is difficult because of the wide variety of different assessment scales used in community based studies. ${ }^{1}$ Individuals in our sample were self selected and generally physically fit whereas most studies looking at the prevalence of anxiety and depression in the community have used a general population with higher levels of co-morbidity. Individuals at high risk of influenza (those with chronic illness or people living in residential and nursing homes) were not eligible for this study and may have higher levels of anxiety or depression than was seen in this sample, as may those individuals who declined consent.

Reporting bias could have occurred since the baseline questionnaires were completed at the general practice with researchers present, thus participants may have been inclined to answer more positively. It could also be speculated that participation in the study might have enhanced people's lives to some degree.

A significant rise in mean depression scores was seen after two months. This could be related to the rise in general morbidity seen in older individuals during the winter months, or that the people in this study were more likely to complete the two, four, and six month postal questionnaires more accurately and truthfully at home. This is borne out by the significant decrease in mean score variation after two months (fig 2).

Individuals with anxiety or depression (HADS score $\geqslant 8$ ) were significantly more likely to complain of systemic side effects after vaccination. This is an important finding since concern about side effects is often quoted as a reason for not being vaccinated, ${ }^{90}$ and people with anxiety or depression may contribute to the incidence of systemic side effects reported in clinical trials. In randomised controlled trials, it has been shown that there is no significant difference in the incidence of systemic side effects between people administered influenza vaccine and those given placebo injections. ${ }^{11}{ }^{12}$

The EQ-5D quality of life score was significantly reduced in individuals with both anxiety and depression (HADS score $\geqslant 8$. Additionally people scoring 8 or more on either the anxiety or depression subscale had a significantly lower mean Barthel score. Thus it appears that anxiety and depression impact considerably on quality of life and are associated with a loss of functional ability.

It is reassuring that individuals were not significantly more anxious at the start of the study compared to other time periods (fig 1). We can therefore assume that the process of volunteering for and participation in a clinical trial has not caused undue anxiety and distress, which could have reduced some of the health benefits that may be shown to occur in the study due to the vaccination programme.

Figures 1 and 2 relate to individuals who returned questionnaires and answered all of the questions on either subscale and may not be truly representative of the whole sample. However no significant association was seen between non-return of questionnaire at any two month period and baseline anxiety or depression score. It therefore seems unlikely that individuals not returning questionnaires were any more anxious or depressed than the other individuals in the study.

In conclusion, when planning future clinical trials the effect of anxiety and depression on older participants must be remembered. If outcome measures such as quality of life, functional ability and perceived side effects are important, care must be taken to ensure that anxiety and depression do not affect results more than the intervention under study.

\section{ACKNOWLEDGEMENTS}

Miss Amanda Platt was responsible for managing the database and assisting with the administration, collection, and input of data. 
This study was funded by the National Coordinating Centre for Health Technology Assessment (Project No: 97/20/06). The views and opinions expressed by the authors in this report do not necessarily reflect those of the NHS Executive.

\section{Authors' affiliations}

S J Allsup, M A Gosney, Department of Geriatric Medicine, University of Liverpool, Liverpool, UK

\section{REFERENCES}

1 Beekman AT, Copeland JR, Prince M. Review of community prevalence of depression in later life. BrJ Psychiatry 1999;174:307-11.

2 Manela $M$, Katona $C$, Livingston $G$. How common are the anxiety disorders in old age? Int J Geriatr Psychiatry 1996;1 1:65-70.

3 de Beurs E, Beekman AT, van Balkom AJ, et al. Consequences of anxiety in older persons: its effect on disability, well-being and use of health services. Psychol Med 1999;29:583-93.

4 Wells KB, Stewart A, Hays RD, et al. The functioning and well being of depressed patients. Results from the Medical Outcomes Study. JAMA 1989:262:914-9.
5 Department of Health. Influenza immunisation: extension of current policy to include all those aged 75 years and over. London: Stationery Office, 1998.

6 Zigmond AS, Snaith RP. The hospital anxiety and depression scale. Acta Psychiatr Scand 1983:67:361-70.

7 Herrmann C. International experiences with the hospital anxiety and depression scale-a review of validation data and clinical results. J Psychosom Res 1997;42:17-41.

8 Silverstone PH. Poor efficacy of the hospital anxiety and depression scale in the diagnosis of major depressive disorder in both medical and psychiatric patients. J Psychosom Res 1994;38:441-50.

9 van Essen GA, Kuyvenhoven MM, de Melker RA. Why do healthy elderly people fail to comply with vaccination? Age Ageing 1997;26:275-9.

10 Gupta A, Makinde K, Morris G, et al. Influenza immunisation coverage in older hospitalised patients during winter 1998-99 in Carmarthenshire UK. Age Ageing 2000;29:211-3.

11 Govaert TM, Dinant GJ, Aretz K, et al. Adverse reactions to influenza vaccination in elderly people: randomised double blind placebo controlled trial. BM 1993;307:988-90.

12 Allsup SJ, Gosney M, Regan M, et al. Side effects of influenza vaccination in healthy older people: a randomised single blind placebo controlled trial. Gerontology 2001;47:311-4. 